\title{
HERBICIDAS NO MANEJO DE INVASORAS EM PASTAGEM DE Brachiaria brizantha CV MARANDU, NO MATO GROSSO, BRASIL
}

\author{
Herbicides in the pasture weed management of Brachiaria \\ brizantha cv Marandu, in Mato Grosso, Brazil
}

\footnotetext{
Thamaturgo Guimarães Castro Junior ${ }^{1}$, Andréa C. Fernandes ${ }^{2}$, Paulo Rossi Junior ${ }^{3}$

${ }^{1}$ Engenheiro Agrônomo, M. Sc., Consultoria Agronômica Castro. Castro, PR - Brasil. e-mail: turgos_castro@hotmail.com

2 Engenheira Agrônoma, Castilho Daitschman \& Cia. Ltda., Curitiba, PR - Brasil. e-mail: agro.fernandes@gmail.com

${ }^{3}$ Zootecnista, Dr., Professor do Departamento de Zootecnia da UFPR, Curitiba, PR - Brasil. e-mail: parossi@ufpr.br
}

\begin{abstract}
Resumo
Visando avaliar formulações e doses de herbicidas para controle de invasoras em pastagem de Brachiaria brizantha cv Marandu, foi conduzido um experimento, em Nova Canaã do Norte, Mato Grosso. O ensaio consistiu de sete misturas de herbicidas, em diferentes doses, sendo os tratamentos: $\mathrm{A}=$ Flumioxazin + Metsulfuron $\left(30 \mathrm{~g}+10 \mathrm{~g}\right.$ p.c.ha $\left.{ }^{-1}\right) ; \mathrm{B}=$ Flumioxazin + Metsulfuron $(20 \mathrm{~g}+10$ g p.c.ha $\left.{ }^{-1}\right) ; \mathrm{C}=$ Flumioxazin + Metsulfuron $+2,4-\mathrm{D}\left(20 \mathrm{~g}+10 \mathrm{~g}+300 \mathrm{~mL}\right.$ p.c.ha $\left.{ }^{-1}\right) ; \mathrm{D}=$ Metsulfuron + 2,4-D (10 g + $500 \mathrm{~mL}$ p.c.ha $\left.{ }^{-1}\right) ; \mathrm{E}=$ Metsulfuron + 2,4-D $(10 \mathrm{~g}+300 \mathrm{~mL}$ p.c.ha $\left.{ }^{1}\right), \mathrm{F}=$ Flumioxazin $+2,4-\mathrm{D}\left(30 \mathrm{~g}+300 \mathrm{~mL}\right.$ p.c.ha $\left.{ }^{-1}\right)$ e $\mathrm{G}=$ Flumioxazin $+2,4-\mathrm{D}(30 \mathrm{~g}+500 \mathrm{~mL}$ p.c.ha $\left.{ }^{-1}\right)$. O delineamento experimental foi em blocos ao acaso com quatro repetições. Os produtos foram aplicados sobre as invasoras de folhas largas. O controle foi avaliado visualmente, aos 15, 30, 45 e 60 dias após o tratamento. As avaliações visuais indicaram que, para as espécies Vernonia sp., Arrabidea sp. e Machaerium aculeatum, os tratamentos mais eficazes foram A, D e G, com controle total até os 60 dias após a aplicação. No caso das demais espécies, todos os tratamentos avaliados apresentaram controle total até os 60 dias após a aplicação. Conclui-se que os herbicidas avaliados podem ser alternativas para o controle de invasoras em pastagens de Brachiaria brizantha cv Marandu e podem proporcionar redução de custos aos pecuaristas.
\end{abstract}

Palavras-chave: Plantas infestantes; Pastagem tropical; Brachiaria brizantha; Herbicidas. 


\begin{abstract}
Aiming to evaluate herbicides formulations and doses for weeds control in Brachiaria brizantha cv Marandu pasture an experiment was conducted in Nova Canaã do Norte, Mato Grosso, Brazil. The research trial consisted of seven herbicides mixtures, in different doses, being the treatments: $=$ Flumioxazin + Metsulfuron $\left(30 \mathrm{~g}+10 \mathrm{~g}\right.$ c.p.ha $\left.{ }^{-1}\right) ; B=$ Flumioxazin + Metsulfuron $(20 \mathrm{~g}+10 \mathrm{~g}$ p.c.ha-1 $) ; C=$ Flumioxazin + Metsulfuron $+2.4-D(20 \mathrm{~g}+10 \mathrm{~g}+300 \mathrm{~mL}$ c.p.ha $\left.{ }^{1}\right) ; D=$ Metsulfuron $+2.4-D\left(10 g+500 m L\right.$ c.p.ha $\left.a^{-1}\right) ; E=$ Metsulfuron $+2.4-D(10 g+300 m L$ c.p.h $\left.\mathrm{a}^{-1}\right), F=$ Flumioxazin $+2.4-D\left(30 \mathrm{~g}+300 \mathrm{~mL}\right.$ c.p.ha $\left.{ }^{-1}\right)$ and $\mathrm{G}=$ Flumioxazin $+2.4-D(30$ $500 \mathrm{~g}+m \mathrm{~L}$ c.p.h $\left.\mathrm{a}^{-1}\right)$. The experimental design was organized in randomized blocks with four replications. The herbicides were applied on the broadleaf species. The weeds control was evaluated by visual analysis at 15, 30, 45 and 60 DAT (days after the treatment). The most efficient treatments were $A, D$ and $G$, with $100 \%$ control, up to 60 days after the treatment for the species Vernonia sp. (Bake fish), Arrabidea sp. (Liana) and Machaerium aculeatum (Mamica). All treatments killed $100 \%$ of the other weed species up to 60 days after application. The used herbicides can be an alternative for weed control in Brachiaria brizantha cv Marandu pastures and can provide cost reduction to the cattle farmers.
\end{abstract}

Keywords: Weeds; Tropical pasture; Brachiaria brizantha; Herbicides.

\title{
INTRODUÇÃO
}

Todo o conflito de competitividade das pastagens tropicais gira em torno das plantas daninhas, fertilidade dos solos e nutrição de plantas, além do manejo dos piquetes. Os manejos diversificados pelos pecuaristas ocasionam alterações no rendimento da pastagem, pelos métodos utilizados, como: roçadas, fogo, grade e uso dos herbicidas.

As poáceas do gênero Brachiaria são originárias da África e largamente utilizadas na América tropical. Existem cerca de 40 milhões de hectares cobertos por pastagens de braquiárias, especialmente no Brasil Central e na Amazônia (MACEDO, 1995). A expansão de áreas de pastagens de braquiária ocorreu por dois motivos: adaptação às condições de solo e adaptação ao clima do Brasil Central e da Amazônia (SANTOS FILHO, 1990). Porém, as pastagens de braquiárias, apesar de seu bom estabelecimento, quando manejadas inadequadamente, acabam por apresentar grande presença de plantas invasoras.

Os produtos recomendados pela pesquisa e distribuídos pelo mercado nacional têm encontrado algumas dificuldades de controle das plantas invasoras. O custo dos herbicidas normalmente utilizados vem se mantendo elevado e a eficácia no controle tem deixado a desejar. Isso ocasiona aumento de custos, em função das doses aplicadas por hectare, preocupação que vem acentuando-se nas pastagens tropicais comumente utilizadas no setor da produção de carne.

A necessidade de ensaios com herbicidas, em nível de campo, surgiu diante do aumento da intensidade das infestações de plantas daninhas, com sensíveis prejuízos econômicos, em toda a região norte do Mato Grosso. O uso intenso que se faz dos herbicidas em pastagens, as doses dos ingredientes ativos indicados e a provável existência da resistência das plantas infestantes às moléculas existentes no mercado, bem como a indiferença dos pecuaristas em receber consultorias agronômicas para planejar o manejo dos agrotóxicos em pastagens, melhorando o rendimento econômico da atividade, justificam, hoje, pesquisas nesta área. 
A infestação de plantas daninhas em pastagens não ocorre como fenômeno isolado e deve ser analisada de forma sistêmica. O surgimento da proliferação de invasoras, tanto em pastagens naturais, quanto cultivadas, é uma resposta ecológica e está relacionada ao manejo aplicado. A presença de plantas daninhas em pastagens é sempre reflexo de práticas anteriores, onde o manejo inapropriado fez com que as espécies desejáveis se tornassem menos competitivas, abrindo espaço para as indesejáveis (KEMP; KING, 2001).

Muitas plantas infestantes não poderiam ser consideradas altamente competitivas, pois não teriam habilidade de invadir e persistir em uma pastagem bem estabelecida e manejada. $\mathrm{O}$ aparente sucesso de muitas plantas daninhas em pastagem está diretamente ligado à capacidade que elas têm para adaptar-se às práticas comuns, como a queima, o pastejo e a roçada (DIAS FILHO, 1990).

Na Região Amazônica, a ocorrência de plantas infestantes é considerada como o mais sério problema de ordem biológica a ser enfrentado pelos pecuaristas, além de ser o seu controle um dos mais elevados componentes do custo de produção das propriedades. Por isso, o estabelecimento de estratégias que possibilitem reduzir o grau de infestação das pastagens, mantendo a longevidade produtiva em longo prazo, em níveis aceitáveis de infestação, é fundamental para a sobrevivência da atividade na região e para a sua lucratividade (EMBRAPA, 2006).

O controle químico exerce ação corretiva sobre o problema, permitindo o desenvolvimento da forrageira e seu aproveitamento pelos animais. Porém, a manutenção da fertilidade, pressão de pastejo, controle das pragas e lotes homogêneos dos animais permitem o correto manejo da pastagem, reduzindo a competitividade das plantas daninhas.

Diante do exposto, dada a importância que o problema com plantas daninhas possui nas pastagens tropicais, o presente trabalho teve o objetivo de avaliar diferentes misturas de herbicidas para o controle químico de plantas daninhas em pastagem de Brachiaria brizantha cv Marandu, no norte do estado do Mato Grosso.

\section{MATERIAL E MÉTODOS}

O ensaio foi desenvolvido na Fazenda Mabagra Agropastoril Ltda., rodovia MT 320, km 88, município de Nova Canaã do Norte, estado do Mato Grosso, clima tropical Aw segundo classificação de Köepen, com estação seca definida, precipitações pluviais acima dos $2000 \mathrm{~mm}$ anuais, temperaturas médias anuais de $28^{\circ} \mathrm{C}$, solos com predominância de latossolo vermelho amarelo álico, cambissolo e areias quartzozas (MAPA, 2001).

Os tratamentos herbicidas utilizados são apresentados na tabela 1 e foram determinados em função do conhecimento das aplicações de manejo das áreas de soja e milho para plantio direto. A eficiência de atuação dos produtos nas plantas infestantes e o baixo custo dos produtos, nas dosagens normalmente utilizadas, foram os critérios para definição dos tratamentos.

A área do ensaio, com pastagem de Brachiaria brizantha cv Marandu, encontrava-se com alta infestação das plantas daninhas, pois no ano anterior, 2005, o proprietário não teve recursos financeiros para aplicar herbicidas em 1.800,0 ha de pastagens com Brachiaria. Neste período, foi utilizada a roçada como forma de manejo das invasoras, porém sem sucesso efetivo. Por ocasião do ensaio, as plantas infestantes, perenizadas na pastagem, apresentavam-se com excelente desenvolvimento vegetativo.

O ensaio foi instalado 06/10/2006 e concluído 06/12/2006. A aplicação dos tratamentos foi realizada usando-se pulverizador costal, com bicos tipo leque $110^{\circ} 03$ e volume de calda de 400 litros por hectare. A pulverização foi realizada respeitando-se as condições climáticas ideais de umidade relativa do ar, temperatura e velocidade do vento. 
TABELA 1 - Tratamentos utilizados para o controle das plantas daninhas, aplicados em pós-emergência, em pastagem de Brachiaria brizantha degradada, marca comercial, doses do ingrediente ativo (i.a.), produto comercial (p.c), Fazenda Mabagra Ltda., Nova Canaã do Norte, MT, 2006

Table 1 - Treatments used for weeds control, applied after emergency, in Brachiaria brizantha degraded pasture; commercial name, active ingredient (a.i.) doses, commercial product (c.p), Mabagra Ltda. Farm, Nova Canaã do Norte, Mato Grosso, 2006

\begin{tabular}{|c|c|c|c|}
\hline $\begin{array}{l}\text { Tratamentos } \\
\text { (Ingrediente Ativo) }\end{array}$ & $\begin{array}{l}\text { Marca } \\
\text { Comercial }\end{array}$ & $\begin{array}{l}\text { Equivalente } \\
\text { ácido }\left(\mathrm{g} \cdot \mathrm{ha}^{-1}\right)\end{array}$ & $\begin{array}{l}\text { Produto Comercial } \\
\left(\mathrm{g} \cdot \mathrm{ha}^{-1} \text { ou } \mathrm{mL} \cdot \mathrm{ha}^{-1}\right)\end{array}$ \\
\hline A. Flumioxazin + Metsulfuron & Flumyzin $500+$ Ally & $15,0 \mathrm{~g}+6,0 \mathrm{~g}$ & $30 g+10 g$ \\
\hline B. Flumioxazin + Metsulfuron & Flumyzin $500+$ Ally & $10,0 \mathrm{~g}+6,0 \mathrm{~g}$ & $20 g+10 g$ \\
\hline C. Flumioxazin + Metsulfuron $+2,4-D$ & $\begin{array}{l}\text { Flumyzin } 500+ \\
\text { Ally }+ \text { U46 D Fluid }\end{array}$ & $10,0 g+6,0 g+108,0 g$ & $20 \mathrm{~g}+10 \mathrm{~g}+300 \mathrm{~mL}$ \\
\hline D. Metsulfuron + 2,4-D & Ally + U46 D Fluid & $6,0 \mathrm{~g}+180,0 \mathrm{~g}$ & $10 \mathrm{~g}+500 \mathrm{~mL}$ \\
\hline E. Metsulfuron + 2,4-D & Ally + U46 D Fluid & $6,0 \mathrm{~g}+108,0 \mathrm{~g}$ & $10 \mathrm{~g}+300 \mathrm{~mL}$ \\
\hline F. Flumioxazin $+2,4-\mathrm{D}$ & $\begin{array}{l}\text { Flumyzin } 500+ \\
\text { U46 D Fluid }\end{array}$ & $15,0 \mathrm{~g}+108,0 \mathrm{~g}$ & $30 \mathrm{~g}+300 \mathrm{~mL}$ \\
\hline G. Flumioxazin + 2,4-D & $\begin{array}{l}\text { Flumyzin } 500+ \\
\text { U46 D Fluid }\end{array}$ & $15,0 \mathrm{~g}+180,0 \mathrm{~g}$ & $30 \mathrm{~g}+500 \mathrm{~mL}$ \\
\hline
\end{tabular}

O delineamento experimental foi em blocos ao acaso, com sete tratamentos e quatro repetições. Cada parcela possuía 3,0 metros de largura por 4,0 metros de comprimento.

As espécies presentes na área do ensaio foram: Assa Peixe (Vernonia spp), Cipó (Arrabidea sp), Joá de capote (Nicandra physaloides), Mamica-de-porca (Machaerium aculeatum), Guanxuma (Sida rhombifolia), Leiteiro (Euphorbia heterophylla), Arranha-gato (Acacia plumosa), Buva (Conyza bonariensis), Fedegoso (Senna obtusifolia), Mentrasto (Ageratum conyzoides), Carrapicho-beiço-de-boi (Desmodium purpureum), Caruru (Amaranthus viridis), Cheirosa (Hyptis suaveolens) e Malva (Sida cordifolia).

A avaliação do controle das plantas daninhas foi realizada por meio de atribuição visual de porcentuais de controle, de acordo com a escala da Asociación Latinoamericana de Malezas (ALAM, 1974), realizada aos 15, 30, 45 e 60 dias após a aplicação dos tratamentos (DAT).

De acordo com essa escala, entre zero e $40 \%$ de controle, este é considerado como nenhum a péssimo controle, entre 41 e $60 \%$, controle regular; entre 61 e $70 \%$, controle suficiente; entre 71 e $80 \%$, bom controle; entre 81 e $90 \%$, controle muito bom e entre 91 e $100 \%$, controle excelente das plantas infestantes.

A análise estatística consistiu em análise de variância e comparação de médias pelo Teste Tukey. A análise estatística foi realizada por meio do software MSTAT v.2.10 (Michigan State University).

\section{RESULTADOS E DISCUSSÃO}

\section{A. Controle de Assa Peixe (Vernonia spp)}

$\mathrm{Na}$ Tabela 2 encontram-se os resultados para o controle de Assa Peixe (Vernonia spp), onde se percebe que as misturas de Flumyzin $500+$ Ally $\left(30 \mathrm{~g}+10\right.$ g.p.c.ha $\left.{ }^{-1}\right)$, Ally + U46-D $(10 \mathrm{~g}+500 \mathrm{~mL}$ p.c.ha $\left.{ }^{-1}\right)$ e Flumyzin $500+$ U46-D $\left(30 \mathrm{~g}+500 \mathrm{~mL}\right.$ p.c.ha $\left.{ }^{-1}\right)$ exerceram controle total sobre a espécie estudada, até os 60 dias após a aplicação do tratamento, e foram estatisticamente superiores aos demais. 
Herbicidas no manejo de invasoras em pastagens de

Brachiaria brizantha cv Marandu, no Mato Grosso, Brasil

TABELA 2 - Controle de Assa Peixe (Vernonia spp), em porcentagem, aos 15, 30, 45 e 60 dias após os tratamentos (DAT), com aplicação de herbicidas em pós-emergência na pastagem de

Brachiaria brizantha, Fazenda Mabagra Ltda., Nova Canaã do Norte, MT, 2006

Table 2 - Assa-Peixe (Vernonia spp) Control (\%), at 15, 30, 45 and 60 days after treatments application (DAT), with herbicides after emergency in a Brachiaria brizantha pasture, at the Mabagra Ltda. Farm, Nova Canaã do Norte, Mato Grosso, 2006

\begin{tabular}{lllll}
\hline Tratamentos & 15 DAT & 30 DAT & 45 DAT & 60 DAT \\
\hline A. Flumyzin 500 + Ally & $100,0 \mathrm{a}$ & $100,0 \mathrm{a}$ & $100,0 \mathrm{a}$ & $100,0 \mathrm{a}$ \\
B. Flumyzin 500 + Ally & $72,5 \mathrm{~b}$ & $77,5 \mathrm{~b}$ & $82,5 \mathrm{~b}$ & $87,5 \mathrm{~b}$ \\
C. Flumyzin 500 + Ally + U46-D Fluid & $65,0 \mathrm{~b}$ & $70,0 \mathrm{c}$ & $70,0 \mathrm{c}$ & $75,0 \mathrm{c}$ \\
D. Ally + U46-D Fluid & $100,0 \mathrm{a}$ & $100,0 \mathrm{a}$ & $100,0 \mathrm{a}$ & $100,0 \mathrm{a}$ \\
E. Ally + U46-D Fluid & $72,5 \mathrm{~b}$ & $77,5 \mathrm{~b}$ & $82,5 \mathrm{~b}$ & $87,5 \mathrm{~b}$ \\
F. Flumyzin 500 + U46-D Fluid & $67,5 \mathrm{~b}$ & $77,5 \mathrm{~b}$ & $85,0 \mathrm{~b}$ & $90,0 \mathrm{~b}$ \\
G. Flumyzin 500 + U46-D Fluid & $100,0 \mathrm{a}$ & $100,0 \mathrm{a}$ & $100,0 \mathrm{a}$ & $100,0 \mathrm{a}$ \\
Coeficiente de Variação & $5,06 \%$ & $3,73 \%$ & $3,96 \%$ & $3,84 \%$ \\
\hline
\end{tabular}

Letras diferentes na vertical diferem entre si pelo teste de Tukey a $5 \%$ de probabilidade.

\section{B. Controle de Cipó (Arrabidea sp)}

De acordo com os dados constantes na Tabela 3, os tratamentos: Flumyzin $500+$ Ally (30 $\mathrm{g}+10$ g.p.c.ha $\left.{ }^{-1}\right)$, Ally + U46-D $\left(10 \mathrm{~g}+500 \mathrm{~mL}\right.$ p.c.ha $\left.{ }^{-1}\right)$, e Flumyzin $500+$ U46-D $(30 \mathrm{~g}+500 \mathrm{~mL}$ p.c.ha ${ }^{-1}$ ), proporcionaram controle excelente desta planta daninha, até os 60 dias após o tratamento, e foram superiores aos demais tratamentos. O tratamento Flumyzin $500+$ Ally + U46-D Fluid $(20 \mathrm{~g}+$ $10 \mathrm{~g}+300 \mathrm{~mL}$ p.c.ha ${ }^{-1}$ ), embora tenha sido estatisticamente diferente dos três citados anteriormente, também apresentou controle excelente da espécie considerada.

TABELA 3 - Controle de Cipó (Arrabidea sp), em porcentagem, aos 15, 30, 45 e 60 dias após o tratamento (DAT), com aplicação de herbicidas em pós-emergência na pastagem de Brachiaria brizantha, Fazenda Mabagra Ltda., Nova Canaã do Norte, MT, 2006

Table 3 - Liana (Arrabidea sp.) control (\%), at 15, 30, 45 and 60 days after treatment application (DAT), with herbicides after emergency in a Brachiaria brizantha pasture, at the Mabagra Ltda. Farm, Nova Canaã do Norte, Mato Grosso, 2006

\begin{tabular}{lllll}
\hline Tratamentos & 15 DAT & 30 DAT & 45 DAT & 60 DAT \\
\hline A. Flumyzin 500 + Ally & $100,0 \mathrm{a}$ & $100,0 \mathrm{a}$ & $100,0 \mathrm{a}$ & $100,0 \mathrm{a}$ \\
B. Flumyzin 500 + Ally & $70,0 \mathrm{~b}$ & $77,0 \mathrm{~b}$ & $85,0 \mathrm{~b}$ & $90,0 \mathrm{~b}$ \\
C. Flumyzin 500 + Ally + U46-D Fluid & $85,5 \mathrm{~b}$ & $90,0 \mathrm{c}$ & $90,0 \mathrm{c}$ & $92,5 \mathrm{c}$ \\
D. Ally + U46-D Fluid & $100,0 \mathrm{a}$ & $100,0 \mathrm{a}$ & $100,0 \mathrm{a}$ & $100,0 \mathrm{a}$ \\
E. Ally + U46-D Fluid & $67,5 \mathrm{~b}$ & $75,0 \mathrm{~b}$ & $87,5 \mathrm{~b}$ & $90,0 \mathrm{~b}$ \\
F. Flumyzin 500 + U46-D Fluid & $62,5 \mathrm{~b}$ & $67,5 \mathrm{~b}$ & $72,5 \mathrm{c}$ & $77,5 \mathrm{~b}$ \\
G. Flumyzin 500 + U46-D Fluid & $80,0 \mathrm{a}$ & $90,0 \mathrm{a}$ & $90,0 \mathrm{~b}$ & $100,0 \mathrm{a}$ \\
Coeficiente de Variação & $5,92 \%$ & $4,24 \%$ & $4,05 \%$ & $4,55 \%$ \\
\hline
\end{tabular}

Letras diferentes na vertical diferem entre si pelo teste de Tukey a $5 \%$ de probabilidade.

\section{Controle de Joá de capote (Nicandra physaloides)}

Para o Joá de capote, com dados constando na Tabela 4, só houve diferenças entre os tratamentos na avaliação aos 15 dias depois do tratamento. Nas demais avaliações, todos os tratamentos testados apresentaram excelente controle da planta daninha, e o controle foi mantido até os 60 dias após ter sido realizada a aplicação. 
TABELA 4 - Controle de Joá de capote (Nicandra physaloides), em porcentagem, aos 15, 30, 45 e 60 dias após o tratamento (DAT), com aplicação de herbicidas em pós-emergência na pastagem de Brachiaria brizantha, Fazenda Mabagra Ltda., Nova Canaã do Norte, MT, 2006

Table 4 - Joá de capote (Nicandra physaloides) control (\%), at 15, 30, 45 and 60 days after treatment application (DAT), with herbicides after emergency in a Brachiaria brizantha pasture, at the Mabagra Ltda. Farm, Nova Canaã do Norte, Mato Grosso, 2006

\begin{tabular}{lcccc}
\hline Tratamentos & 15 DAT & 30 DAT & 45 DAT & 60 DAT \\
\hline A. Flumyzin 500 + Ally & $100,0 \mathrm{a}$ & 100,0 & 100,0 & 100,0 \\
B. Flumyzin 500 + Ally & $82,5 \mathrm{~b}$ & $90,0 \mathrm{~b}$ & 90,0 & 97,5 \\
C. Flumyzin 500 + Ally + U46-D Fluid & $82,5 \mathrm{~b}$ & 90,0 & 90,0 & 97,5 \\
D. Ally + U46-D Fluid & $10,0 \mathrm{a}$ & 10,0 & 10,0 & 10,0 \\
E. Ally + U46-D Fluid & $82,5 \mathrm{~b}$ & 90,0 & 90,0 & 100,0 \\
F. Flumyzin 500 + U46-D Fluid & $82,5 \mathrm{~b}$ & 90,5 & 90,0 & 100,0 \\
G. Flumyzin 500 + U46-D Fluid & $100,0 \mathrm{a}$ & 100,0 & 100,0 & 100,0 \\
Coeficiente de Variação & $2,97 \%$ & - & - & - \\
\hline
\end{tabular}

Letras diferentes na vertical diferem entre si pelo teste de Tukey a $5 \%$ de probabilidade.

\section{Controle de Mamica-de-porca (Machaerium aculeatum)}

Para a Mamica-de-porca (Tabela 5), os tratamentos: Flumyzin $500+$ Ally (30 g + 10 g.p.c.ha $\left.{ }^{-1}\right)$; Ally + U46-D (10 g + $500 \mathrm{~mL}$ p.c.ha $\left.{ }^{-1}\right)$; e Flumyzin $500+$ U46-D $\left(30 \mathrm{~g}+500 \mathrm{~mL}\right.$ p.c.ha $\left.{ }^{-1}\right)$ proporcionaram controle excelente desta planta daninha, até os 60 dias após o tratamento.

TABELA 5 - Controle de Mamica-de-porca (Machaerium aculeatum), em porcentagem, aos 15, 30, 45 e 60 dias após o tratamento (DAT), com aplicação de herbicidas em pós-emergência na pastagem de Brachiaria brizantha, Fazenda Mabagra Ltda., Nova Canaã do Norte, MT, 2006

Table 5 - Mamica-de-porca (Machaerium aculeatum) control (\%), at 15, 30, 45 and 60 days after treatment application (DAT), with herbicides after emergency in a Brachiaria brizantha pasture, at the Mabagra Ltda. Farm, Nova Canaã do Norte, Mato Grosso, 2006

\begin{tabular}{lllll}
\hline Tratamentos & 15 DAT & 30 DAT & 45 DAT & 60 DAT \\
\hline A. Flumyzin 500 + Ally & $100,0 \mathrm{a}$ & 100,0 & $100,0 \mathrm{a}$ & $100,0 \mathrm{a}$ \\
B. Flumyzin 500 + Ally & $70,0 \mathrm{bc}$ & $77,5 \mathrm{~b}$ & $77,5 \mathrm{~b}$ & $85,0 \mathrm{~b}$ \\
C. Flumyzin 500 + Ally + U46-D Fluid & $62,5 \mathrm{c}$ & $67,5 \mathrm{c}$ & $70,0 \mathrm{c}$ & $75,0 \mathrm{c}$ \\
D. Ally + U46-D Fluid & $100,0 \mathrm{a}$ & $100,0 \mathrm{a}$ & $100,0 \mathrm{a}$ & $100,0 \mathrm{a}$ \\
E. Ally + U46-D Fluid & $70,0 \mathrm{bc}$ & $77,5 \mathrm{~b}$ & $82,5 \mathrm{~b}$ & $90,0 \mathrm{~b}$ \\
F. Flumyzin 500 + U46-D Fluid & $72,5 \mathrm{~b}$ & $80,0 \mathrm{~b}$ & $80,0 \mathrm{~b}$ & $87,5 \mathrm{~b}$ \\
G. Flumyzin 500 + U46-D Fluid & $100,0 \mathrm{a}$ & $100,0 \mathrm{a}$ & $100,0 \mathrm{a}$ & $100,0 \mathrm{a}$ \\
Coeficiente de Variação & $4,27 \%$ & $3,11 \%$ & $3,31 \%$ & $3,46 \%$ \\
\hline
\end{tabular}

Letras diferentes na vertical diferem entre si pelo teste de Tukey a $5 \%$ de probabilidade.

\section{E. Controle de outras espécies}

No caso das espécies: Arranha-gato (Acacia plumosa), Buva (Conyza bonariensis), Fedegoso (Senna obtusifolia), Mentrasto (Ageratum conyzoides), Carrapicho-beiço-de-boi (Desmodium purpureum), Caruru (Amaranthus viridis), Cheirosa (Hyptis suaveolens), Guanxuma (Sida rhombifolia), Leiteiro (Euphorbia heterophylla), e Malva (Sida cordifolia), na Tabela 6, todos os tratamentos avaliados exerceram controle total, até os 60 dias após a aplicação. Não houve diferenças significativas entre as datas de avaliação, nem tampouco entre os diferentes tratamentos testados. 
Herbicidas no manejo de invasoras em pastagens de

Brachiaria brizantha cv Marandu, no Mato Grosso, Brasil

TABELA 6 - Controle do Arranha-gato (Acacia plumosa), Buva (Conyza bonariensis), Fedegoso (Senna obtusifolia),

Mentrasto (Ageratum conyzoides), Carrapicho-beiço-de-boi (Desmodium purpureum), Caruru (Amaranthus viridis),

Cheirosa (Hyptis suaveolens), Guanxuma (Sida rhombifolia), Leiteiro (Euphorbia heterophylla) e Malva (Sida cordifolia), em porcentagem, aos 15, 30, 45 e 60 dias após o tratamento (DAT), com aplicação de herbicidas em pósemergência na pastagem de Brachiaria brizantha, Fazenda Mabagra Ltda., Nova Canaã do Norte, MT, 2006

Table 6 - Arranha-gato (plumosa Acacia), Buva (Conyza bonariensis), Fedegoso (obtusifolia Senna), Mentrasto (Ageratum conyzoides), Carrapicho-beiço-de-boi (Desmodium purpureum), Caruru (Amaranthus viridis), Cheirosa (Hyptis suaveolens), Guanxuma (Been rhombifolia), Leiteiro (Euphorbia heterophylla) and Malva (Been cordifolia) control (\%), at 15, 30, 45 and 60 days after treatment application (DAT), with herbicides after emergency in a Brachiaria brizantha pasture, at the Mabagra Ltda. Farm, Nova Canaã do Norte, Mato Grosso

\begin{tabular}{lllll}
\hline Tratamentos & 15 DAT & 30 DAT & 45 DAT & 60 DAT \\
\hline A. Flumyzin 500 + Ally & 100,0 & 100,0 & 100,0 & 100,0 \\
B. Flumyzin 500 + Ally & 100,0 & 100,0 & 100,0 & 100,0 \\
C. Flumyzin 500 + Ally + U46-D Fluid & 100,0 & 100,0 & 100,0 & 100,0 \\
D. Ally + U46-D Fluid & 100,0 & 100,0 & 100,0 & 100,0 \\
E. Ally + U46-D Fluid & 100,0 & 100,0 & 100,0 & 100,0 \\
F. Flumyzin 500 + U46-D Fluid & 100,0 & 100,0 & 100,0 & 100,0 \\
G. Flumyzin 500 + U46-D Fluid & 100,0 & 100,0 & 100,0 & 100,0 \\
Coeficiente de Variação & 100,0 & 100,0 & 100,0 & 100,0 \\
\hline
\end{tabular}

TABELA 7 - Controle de espécies infestantes, em porcentagem, aos 60 dias após o tratamento (DAT), com aplicação de herbicidas em pós-emergência na pastagem de Brachiaria brizantha,

Fazenda Mabagra Ltda., Nova Canaã do Norte, MT, 2006

Table 7 - Weeds control (\%), at 60 days after treatment application (DAT), with herbicides after emergency in a Brachiaria brizantha pasture, at the Mabagra Ltda Farm, Nova Canaã do Norte, Mato Grosso, 2006

\begin{tabular}{llllllll}
\hline & \multicolumn{7}{c}{ Tratamentos Aplicados } \\
Espécies Avaliadas & A & B & C & D & E & F & G \\
\hline Vernonia sp & $100,0 \mathrm{a}$ & $87,5 \mathrm{~b}$ & $75,0 \mathrm{c}$ & $100,0 \mathrm{a}$ & $87,5 \mathrm{~b}$ & $90,0 \mathrm{~b}$ & $100,0 \mathrm{a}$ \\
Arrabidea sp & $100,0 \mathrm{a}$ & $90,0 \mathrm{~b}$ & $92,5 \mathrm{ab}$ & $100,0 \mathrm{a}$ & $90,0 \mathrm{~b}$ & $77,5 \mathrm{c}$ & $100,0 \mathrm{a}$ \\
Nicandraphysaloides & 100,0 & 97,5 & 97,5 & 10,00 & 100,0 & 100,0 & 100,0 \\
Machaerium aculeatum & $100,0 \mathrm{a}$ & $85,0 \mathrm{~b}$ & $75,0 \mathrm{c}$ & $100,0 \mathrm{a}$ & $90,0 \mathrm{~b}$ & $87,5 \mathrm{~b}$ & $100,0 \mathrm{a}$ \\
Sida rhombifolia & 100,0 & 100,0 & 100,0 & 100,0 & 100,0 & 100,0 & 100,0 \\
Euphorbia heterophylla & 100,0 & 100,0 & 100,0 & 100,0 & 100,0 & 100,0 & 100,0 \\
Acacia plumosa & 100,0 & 100,0 & 100,0 & 100,0 & 100,0 & 100,0 & 100,0 \\
Conyza bonariensis & 100,0 & 100,0 & 100,0 & 100,0 & 100,0 & 100,0 & 100,0 \\
Senna obtusifolia & 100,0 & 100,0 & 100,0 & 100,0 & 100,0 & 100,0 & 100,0 \\
Ageratum conyzoides & 100,0 & 100,0 & 100,0 & 100,0 & 100,0 & 100,0 & 100,0 \\
Desmodium purpureum & 100,0 & 100,0 & 100,0 & 100,0 & 100,0 & 100,0 & 100,0 \\
Amaranthus viridis & 100,0 & 100,0 & 100,0 & 100,0 & 100,0 & 100,0 & 100,0 \\
Hyptis suaveolens & 100,0 & 100,0 & 100,0 & 100,0 & 100,0 & 100,0 & 100,0 \\
Sida cordifolia & 100,0 & 100,0 & 100,0 & 100,0 & 100,0 & 100,0 & 100,0 \\
\hline
\end{tabular}

Letras diferentes na horizontal diferem entre si pelo teste de Tukey a $5 \%$ de probabilidade.

Tratamentos:

$\mathrm{A}=$ Flumioxazin + Metsulfuron $\left(30 \mathrm{~g}+10 \mathrm{~g}\right.$ p.c.ha $\left.{ }^{-1}\right)$

$\mathrm{B}=$ Flumioxazin + Metsulfuron $\left(20 \mathrm{~g}+10 \mathrm{~g}\right.$ p.c.ha $\left.{ }^{-1}\right)$

$\mathrm{C}=$ Flumioxazin + Metsulfuron $+2,4-\mathrm{D}\left(20 \mathrm{~g}+10 \mathrm{~g}+300 \mathrm{~mL}\right.$ p.c.ha $\left.{ }^{-1}\right) ;$

$\mathrm{D}=$ Metsulfuron $+2,4-\mathrm{D}\left(10 \mathrm{~g}+500 \mathrm{~mL}\right.$ p.c.ha $\left.{ }^{-1}\right)$

$\mathrm{E}=$ Metsulfuron $+2,4-\mathrm{D}\left(10 \mathrm{~g}+300 \mathrm{~mL}\right.$ p.c.ha $\left.{ }^{-1}\right)$

$\mathrm{F}=$ Flumioxazin $+2,4-\mathrm{D}\left(30 \mathrm{~g}+300 \mathrm{~mL}\right.$ p.c.ha $\left.{ }^{-1}\right)$

$\mathrm{G}=$ Flumioxazin $+2,4-\mathrm{D}\left(30 \mathrm{~g}+500 \mathrm{~mL}\right.$ p.c.ha $\left.{ }^{-1}\right)$

Rev. Acad., Ciênc. Agrár. Ambient., Curitiba, v. 6, n. 1, p. 109-118, jan./mar. 2008 
A mistura de Metsulfuron com doses menores de 2,4-D tem sido utilizada como alternativa na cultura do trigo, por exemplo, para prevenir o desenvolvimento da resistência das plantas daninhas aos herbicidas do grupo das sulfoniluréias, caso do Metsulfuron (KLEIN; MARTIN; LYON, 2006). Esta mesma alternativa poderia ser utilizada para o controle de plantas daninhas em pastagem de Brachiaria brizantha $\mathrm{cv}$ Marandu, embora ainda faltem estudos sobre os efeitos e seletividade desta mistura sobre a pastagem.

Brown et al. (2004), em trabalho para investigar a eficácia e a seletividade de misturas de Metsulforon com 2,4-D, Dicamba, Clopyrarid e Fluroxypir para controle de Ipomoea hederacea e Abutilon theophrasti em sorgo, verificaram que a mistura de Metsulfuron com 2,4-D exerceu controle de 95\% sobre Ipomoea hederacea e de $88 \%$ sobre Abutilon theophrasti, em avaliação realizada quatro semanas depois da aplicação. A mistura de Metsulfuron + Dicamba manteve um controle de $84 \%$ e $82 \%$, respectivamente, sobre essas duas plantas, quatro semanas depois da aplicação. Houve sinais de injúrias às plantas de sorgo, principalmente na mistura com 2,4-D, mas a cultura se recuperou.

Dutra, Souza Filho e Mascarenhas (2002), em ensaio para avaliar métodos integrados de controle de infestantes em pastagens de Brachiaria brizantha no Pará (roçada manual, roçada manual mais queima e gradagem, associados ou não ao herbicida $2,4-\mathrm{D}$ nas concentrações de $0,1 \%, 2 \%, 3 \%$, e mais um tratamento com $2 \%$ de herbicida e aplicação de $\mathrm{P}_{2} \mathrm{O}_{5}$ em superfície), concluíram que com a integração de métodos, ou seja, o uso de métodos físicos e o método químico, o controle das espécies infestantes foi efetivo. Sem a associação com o controle químico, os métodos físicos foram incapazes de controlar a infestação. O estudo mostrou que qualquer dos métodos físicos avaliados, associados a uma aplicação de 2,4-D a uma concentração média de $2,2 \%$, proporcionam a máxima resposta da Brachiaria brizantha e supressão das infestantes.

Pereira, Ornelas e Hidalgo (2000), aplicando Metsulfuron (3,0 g i.a.ha ${ }^{-1}$ ) em Brachiaria brizantha cv Marandu, observaram apenas leves sintomas de fitointoxicação na pastagem. Porém, em estudo de seletividade de herbicidas aplicados em pré e pós-emergência, em Panicum maximum cv Tanzânia, Panicum maximum cv Mombaça, Brachiaria brizantha cv Marandu e Brachiaria decumbens cv Basilisk, Alves (2001), concluiu que, para Brachiaria brizantha cv Marandu, em pós-emergência, apenas o Imazethapyr, aplicado nas doses de 50,0 e 100,0 g i.a.ha ${ }^{-1}$, foi seletivo para a pastagem, tanto visualmente, quanto na avaliação de altura de plantas e produção de matéria seca. As moléculas: Metsulfuron (2,0 e 1,0 g i.a.ha $\left.{ }^{-1}\right)$, Chlorimuron (15,0 e 7,5 g i.a.ha $\left.{ }^{-1}\right)$, Nicosulfuron (50,0 e 25,0 g i.a.ha ${ }^{-1}$ ) e Atrazine (3000 e $1500 \mathrm{~g}$ i.a.ha ${ }^{-1}$ ) foram seletivas visualmente, porém não tiveram a seletividade confirmada pela avaliação de altura de plantas e produção de matéria seca. Bentazon $\left(720,0\right.$ e 360,0 g i.a.ha $\left.{ }^{-1}\right)$, Fenoxaprop (60,0 e 30,0 g i.a.ha $\left.{ }^{-1}\right)$ e Ametryne (1250 e 625 g i.a.ha $\left.{ }^{-1}\right)$ não foram seletivos para a Brachiaria brizantha cv Marandu. O Metsulfuron, nas duas dosagens utilizadas, foi seletivo tanto para Panicum maximum cv Tanzânia quanto para Panicum maximum cv Mombaça, mas não para Brachiaria decumbens cv Basilisk e Brachiaria brizantha cv Marandu.

No presente trabalho, a seletividade das misturas utilizadas para a pastagem não foi avaliada, pois o objetivo do trabalho foi avaliar o controle das plantas daninhas. Justifica-se o estudo futuro relativo à seletividade sobre a Brachiaria brizantha cv Marandu, dado o excelente controle exercido sobre as plantas daninhas. O estudo da seletividade justifica-se também pela redução de custos que pode proporcionar ao pecuarista.

\section{CONCLUSÕES}

Os tratamentos utilizados mostraram que opções técnicas são viáveis ao pecuarista, permitindo que exista possibilidade de gerenciamento em todo o processo da produção com avanço das tecnologias e o apoio das consultorias agronômicas. 
Os tratamentos eficientes no controle de Assa peixe (Vernonia sp), Cipó (Arrabidea sp) e Mamica-de-porca (Machaerium aculeatum) foram: Flumyzin $500+$ Ally $\left(30 \mathrm{~g}+10\right.$ g p.c.ha $\left.{ }^{-1}\right)$, Ally + U46D Fluid $\left(10 \mathrm{~g}+500 \mathrm{~mL}\right.$ p.c.ha $\left.{ }^{-1}\right)$, e Flumyzin $500+$ U46-D Fluid $\left(30 \mathrm{~g}+500 \mathrm{~mL}\right.$ p.c.ha $\left.{ }^{-1}\right)$, sendo que os três tratamentos proporcionaram controle considerado excelente, até os 60 dias após a aplicação.

Para as demais espécies citadas no trabalho, todos os tratamentos utilizados proporcionaram controle total e o efeito manteve-se até os 60 dias após a aplicação.

A roçada realizada não teve controle sobre as plantas daninhas, pois o manejo de pressão de corte exercido é intenso no campo, diminuindo a competitividade do crescimento da braquiária, processo do início da degradação total da pastagem.

As misturas testadas apresentaram potencial para tornarem-se novas alternativas no controle químico das plantas infestantes de folhas largas, em pós-emergência, nas pastagens de Bracharia brizantha cv Marandu. Ressalta-se, porém, a necessidade de novos ensaios, utilizando como testemunhas herbicidas normalmente aplicados na região, para fins comparativos.

\section{REFERÊNCIAS}

ALVES, E. Seletividade de herbicidas para gramíneas forrageiras tropicais aplicados em pré e pós-emergência. 2001, 88 f. Dissertação (Mestrado em Agronomia - Área de concentração Agricultura) - Faculdade de Ciências Agronômicas da UNESP, Botucatu. 2001.

ASOCIACIÓN LATINOAMERICANA DE MALEZAS. Recomendaciones sobre unificación de los sistemas de evaluación en ensayos de control de malezas. ALAM, Bogotá, v. 1, p. 35-38, 1974.

BROWN, D. W. et al. Safening grain sorghum injury from metsulfuron with growth regulator herbicides. Weed Science, Canadá, v. 52, n. 3, p. 319-325, 2004.

DIAS FILHO, M. B. Plantas invasoras em pastagens cultivadas na Amazônia: Estratégias de manejo e controle. EMBRAPA, 1990. p. 1-103. n. 52.

DUTRA, S.; SOUZA FILHO, A. P. S.; MASCARENHAS, R. E. B. Controle integrado das espécies invasoras assa-peixe e casadinha em pastagens cultivadas de Paragominas, nordeste paraense. Boletim de Pesquisa e Desenvolvimento, Belém-PA, n. 13, 2002.

EMBRAPA, Manejo de plantas daninhas em pastagens cultivadas. In: HOMMA, A. K. O. et al. Criação de bovinos de corte no estado do Pará. Sistemas de Produção - 3, Belém, dez. 2006. Disponível em: http://sistemasdeproducao.cnptia.embrapa.br/FontesHTML/BovinoCorte/ BovinoCortePara/paginas/manejo.html. Acesso em: 03 jul. 2006.

KEMP, D. R.; KING, W. McG. Plant competition in pastures: implications for management. In: TOW, P. G.; LAZENBY, A. (Ed.). Competition and succession in pastures. New York: CABI Publishing. 2001. p. 85-102.

KLEIN, R. N.; MARTIN, A. R.; LYON, D. J. Annual broadleaf weed control in winter wheat. NebGuide - G1241. Nebraska: University of Nebraska - Lincoln Extension, Institute of Agriculture and Natural Resources. 2006.

MACEDO, M. C. M. Pastagens no ecossistema cerrados: Pesquisa para o desenvolvimento sustentável. In: SIMPÓSIO SOBRE PASTAGENS NOS ECOSSISTEMAS BRASILEIROS: pesquisas para o desenvolvimento sustentável, 1., 1995, Brasília. Anais... Brasília: SBZ. 1995, p. 28-62. 
MAPA de solos do Brasil. (Rio de Janeiro). IBGE, Embrapa Solos. Rio de Janeiro, 2001. Mapa físico. Escala 1: 5.000.000.

PEREIRA, F. A. R.; ORNELAS, A. J.; HIDALGO, E. Avaliação do herbicida Metsulfuron-methyl no controle de plantas daninhas em área de produção de sementes de pastagens. Revista Brasileira de Herbicidas, Passo Fundo, v. 1, n. 2, p. 179-183, 2000.

SANTOS FILHO, L. F. Diagnóstico da situação da produção de sementes de plantas forrageiras no estado de São Paulo. In: ENCONTRO SOBRE PRODUÇÃO DE SEMENTES DE PLANTAS FORRAGEIRAS, 4., 1990, São José do Rio Preto. Anais... São José do Rio preto: Gráfica Só-cópias. 1990. p. 1-14.

Recebido: 02/05/2006

Received: 05/02/2006

Aprovado: 31/10/2007

Approved: 10/31/2007 